\title{
Contributions to the debate on integrality and oral health
}

\section{Nilce Emy Tomita ${ }^{(a)}$ Sigmar de Mello Rode ${ }^{(b)}$}

\footnotetext{
(a) President of ABOPREV - Brazilian Association for Oral Health Promotion.

(b) Scientific Editor of BOR - Brazilian Oral Research.
}

A variety of perspectives on oral health issues guide discussions promoted in the forum historically provided by ABOPREV, with the firm goal of contributing to public policies.

In the yearly 2012 meeting, an "integrality perspective" was selected as the central theme, and served as a basis for reflection. The discussions and reflections produced during the meeting are presented in this special issue of BOR in the form of essays, in an effort to broaden access by the dental community to the subjects addressed.

The meanings usually applied to the term integrality are the coordination of preventive and health care actions, some aspects of organization of health services and the attributes of health care practices. ${ }^{1}$

Concerning the coordination of preventive and health care actions, important aspects are presented in the papers of Ferreira et al. and Moysés. Ferreira $e t$ al. discuss living conditions in a society guided by social inequalities, also regarded as inequities, and further defined as health inequalities that are avoidable, unfair and unnecessary. ${ }^{2}$ The programs are presented as a way of coping with health inequities, making strategic use of technological devices, with a heavy reliance on intersubjectivity and ethics and developing the emancipation ability of individuals.

Moysés highlights that oral diseases, like other health disorders, are socially molded by a relationship known as social gradient. The efforts to reduce oral diseases and inequities show the importance of social determinants and require the establishment of strategies to reduce oral health inequities, including the management of common risk factors from an environmental perspective.

Applications of epidemiology as a tool to gain a better understanding of dental caries as a social phenomenon are discussed in the paper by Frazão. This understanding will enable us to better organize health services, guided by the reduction of income inequalities and public support for advances in systems that distribute resources equally, which in turn may reduce levels of socioeconomic inequities.

Relevant aspects of the organization of health services are discussed in papers by Soares and Chaves. The organization of services is presented by Soares, who addresses the development of oral health policies in Brazil from a critical perspective. The author has observed a progressive expansion of government intervention in health policies, citing as examples:

- the fluoridation of the public water supply and fluoride dentifrices commercially available in Brazil;

- epidemiological oral health surveys;

- the inclusion of oral health services in the Family Health Strategy; and

- the establishment of the National Oral Health Policy, called "Brasil Sorridente" ("Smiling Brazil”).

Chaves selected the oral health of adults as basis for reflection on the health policies that guide—or should guide—the provision of integrated care to this 
segment of the population that has been historically overlooked, and which has led to a significant prevalence of edentulism among Brazilian elderly individuals. The main oral health care models and the technological organization of the work process are discussed, as well as the challenges Brazilian dentistry must overcome to achieve universal access and equity.

Some attributes of health care practices become evident when discussing the care required for an individual experiencing the suffering caused by pain. The oral health care delivered during a meeting between a health professional and a patient with his/her complaint is a crucial step toward integrating health care.

The clinical mechanisms of pain and its perception by the individual, as well as factors predisposing him/ her to pain, are described by Stump and Dalben, culminating in a deeper understanding of pain that will allow therapeutic projects based on pharmacological and biopsychosocial approaches.

Conti et al. address some conditions that may cause painful symptomatology in orofacial structures, such as temporomandibular disorders, providing elements for a clinical intervention while also considering the psychological and physiological aspects that influence the quality of life.

Complementing the management of individuals in pain, Galitesi et al. highlight the importance of a meeting between the individual and the health team, emphasizing the importance of a comprehensive view of the mouth and patient to managing pain, while taking into consideration the vital and emotional aspects of the individual.

Other attributes of health care practices refer to preventive action, put in place in anticipation of the disease experience and based on the wealth of knowledge produced by dental research. This is the theme addressed in the papers by Bönecker and Twetman.

Bönecker et al. selected children as the subject of their practice and demonstrated how oral diseasesdental caries, traumatic lesions and dental erosion-influence the quality of life of childen, placing some at a psychosocial disadvantage.

Twetman discusses the health care of individuals

\section{References}

1. Matos RA. A integralidade na prática (ou sobre a prática da integralidade). Cad. Saude Publica. 2004 Set-Out;20(5):1411-6. who have a wide array of technologies available-hard and soft-hard-to reduce the pathogenic potential of the oral microbiota. The daily use of bacteriotherapy, with emphasis on probiotics, encourages the individual to perform the self-care that allows him/her to modulate dental biofilm, a common risk factor in the main oral diseases.

A special meeting between lecturers and the audience was the focus of a paper that summarizes the discussions held during the master symposium. The discussion between lecturers addressed:

- the transition of dental caries and the role of fluoride dentifrices, framed against an educational backdrop to encourage their conscientious use;

- disease-based practices and the need to develop competences related to multiprofessionality to promote advances in the health care of individuals;

- health education according to the needs of the public; and

- the working world requiring changes in the education world, ultimately leading to the training of health professionals who are aware of systemic aspects.

In a dialogue on the aspects proposed by invited lecturers, some questions by the audience raised important points about the daily routine of health services, multiprofessional team work, training required for dentists for a practice based on integration and the role of community dentistry integrated into the field of individual actions.

This symposium served as a meeting place for individuals with heterogeneous experiences from many places throughout Brazil, who consider health promotion as a point of departure and arrival.

This special issue also includes a paper on the Latin American Oral Health Panel of 2012.

The five-year partnership between ABOPREV and BOR, with the publication of special issues covering the outcomes of the ABOPREV meetings, has been fundamental to update and disseminate state-of-the-art information on oral health, and has become a reference source in the field, evidenced by the popularity of these publications.

2. Whitehead M. The concepts and principles of equity and health. Int J Health Serv. 1992;22(3):429-445. 